\title{
Health-Info-Net, un concept stratégique de la FMH
}

Obtenir et échanger rapidement des données tout en les gérant de manière sûre: voilà qui tient de la quadrature du cercle. A l'avenir, dans leur pratique quotidienne, les médecins seront de plus en plus souvent confrontés à ce dilemme sans issue. Ils le sont d'ailleurs aujourd'hui déjà et nous le sommes tous, souvent sans en avoir conscience.

Les réseaux de données ne cessent de croître, à l'instar de la facilité avec laquelle nous accédons à la technologie internet et de la naïveté avec laquelle nous en usons. Dans ces circonstances, on peut s'attendre à ce qu'éclate une série de scandales, qui ne manqueront pas de porter un coup sérieux à la protection de la personnalité et, partant, des données. Nul doute que cette situation deviendra un thème politique brûlant. Ce n'est pas encore le cas, car, pour l'heure, tant les politiciens que les consommateurs continuent d'agir avec le même laisseraller. Mais de tels désordres pourraient nuire à l'image déjà quelque peu égratignée du corps médical. Il nous appartient donc, dans ce domaine comme dans d'autres, de conduire notre politique professionnelle en fonction d'une stratégie à long terme et de nous rappeler que «gouverner, c'est prévoir».

La faculté de gérer et transmettre des données de façon sûre ne tardera pas à devenir un enjeu professionnel essentiel pour tout praticien. Un nouvel univers s'ouvre, celui de la diffusion de l'information destinée au corps médical et touchant aux domaines les plus variés: formation prégraduée, postgraduée et continue, nouvelles formes de relations patient-médecin, gestion des ressources et gestion en général, sans oublier le contrôle et l'assurance de la qualité. Nombreux sont ceux d'entre nous qui l'ont déjà compris. Et ceux qu'attire irrésistiblement cette nouvelle ruée vers l'or sont moins dangereux que ceux qui entendent mésuser des données disponibles pour en faire un instrument de pouvoir, une démarche illégitime et incontrôlée qui va rarement dans l'intérêt du patient et de son médecin traitant.
Ces mêmes réflexions ont amené la $\mathrm{FMH}$, en 1995 déjà, à développer un réseau informatique spécialement à l'intention des médecins. Désormais connu sous le nom de HIN (HealthInfo-Net), ce dispositif n'a pas vu le jour sans difficultés:

- Le corps médical n'a pas encore pris partout la mesure des difficultés que l'avenir nous réserve.

- La construction de ce réseau électronique, qui exigeait des solutions personnalisées et ne pouvait se satisfaire de matériel prêt à l'emploi, a été marquée par un certain nombre de pannes.

- Le HIN a été financé presque exclusivement par des fonds de la FMH, donc par vos cotisations de membre. Nous ne pouvons malheureusement (ou peut-être heureusement?) pas utiliser vos impôts ou vos primes d'assurance pour régler les dépenses liées à un telle réalisation et aux inévitables bavures qui l'accompagnent ...

Actuellement, plus de cinq mille praticiens recourent aux services du HIN. Cet avantage, nous le devons à l'ensemble du corps médical. Donc à vos cotisations d'une part, mais souvent aussi à vos critiques qui, même si elles n'étaient pas toujours objectives et parfois un peu dures, nous ont toujours permis de progresser.

Les deux sites internet HIN (www.hin.ch) et FMH (www.fmh.ch) vous offrent un bilan complet de tout ce qui a été réalisé à ce jour et de tout ce qui reste à faire. Si nous avons renoncé à publier cette somme de renseignements sous forme écrite, ce n'est pas seulement en raison de son volume. C'est aussi par conviction: nous sommes en effet persuadés qu'en matière de circulation de l'information, une nouvelle ère a définitivement commencé.

Dr Hans Heinrich Brunner 